Provided for non-commercial research and education use. Not for reproduction, distribution or commercial use.

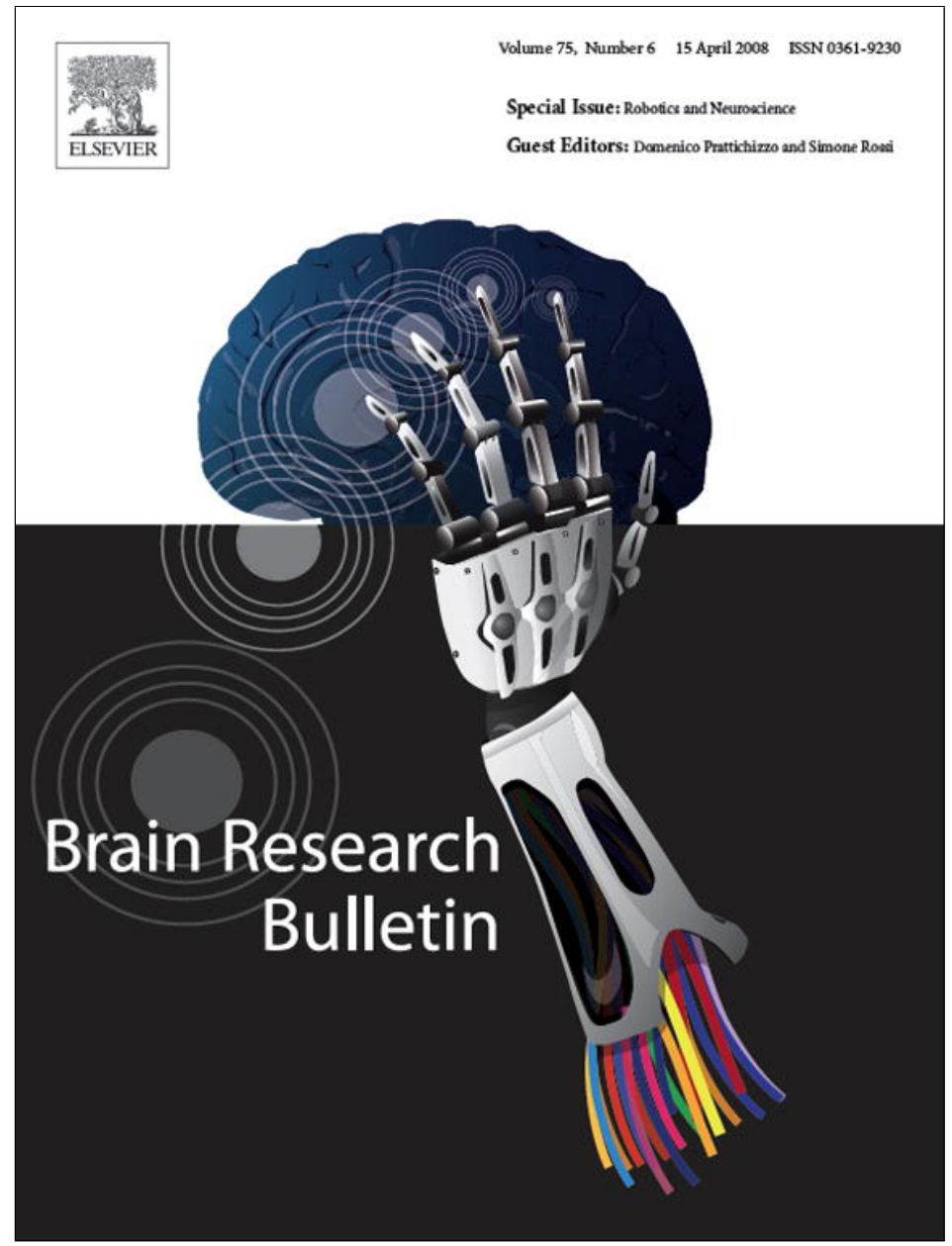

This article appeared in a journal published by Elsevier. The attached copy is furnished to the author for internal non-commercial research and education use, including for instruction at the authors institution and sharing with colleagues.

Other uses, including reproduction and distribution, or selling or licensing copies, or posting to personal, institutional or third party websites are prohibited.

In most cases authors are permitted to post their version of the article (e.g. in Word or Tex form) to their personal website or institutional repository. Authors requiring further information regarding Elsevier's archiving and manuscript policies are encouraged to visit:

http://www.elsevier.com/copyright 
Research report

\title{
Contact forces evoked by transcranial magnetic stimulation of the motor cortex in a multi-finger grasp ${ }^{\text {is }}$
}

\author{
Gabriel Baud-Bovy ${ }^{\mathrm{a}, \mathrm{b}, *}$, Domenico Prattichizzo ${ }^{\mathrm{c}}$, Simone Rossi $^{\mathrm{d}}$ \\ ${ }^{a}$ Faculty of Psychology, Vita-Salute San Raffaele University, Milan, Italy \\ ${ }^{\mathrm{b}}$ IIT Network Research Unit of Molecular Neuroscience, San Raffaele Foundation, Milan, Italy \\ ${ }^{\mathrm{c}}$ Dipartimento di Ingegneria dell'Informazione, Università di Siena, Italy \\ ${ }^{\mathrm{d}}$ Dipartimento di Neuroscienze, Sezione Neurologia, Università di Siena, Italy
}

Available online 5 February 2008

\begin{abstract}
Transcranial magnetic stimulation (TMS) is a tool of choice to study the functionality of the corticospinal pathway. In this study, we used single-pulse TMS at different intensities and during different levels of grasping force, to stimulate the hand area of the left primary motor cortex (M1). We measured, the TMS-evoked forces, or motor evoked forces (MEFs) in a multi-fingered three-point grasp in addition of the conventional motor evoked potentials (MEPs) from the right forearm and intrinsic hand muscles. This paper aims at presenting the viability of this innovative approach and some preliminary results. The timing (i.e., latencies and peak times), amplitudes and directions of the MEF were analyzed. We found that the TMS evoked synergistic increases of the force magnitudes, akin to those observed when participants voluntarily increased the grip force. The MEF sizes and MEP amplitudes increased with TMS intensity in most cases. The grip force (which measures the overall force involved in the grasp) and the net force (which measures the net effect of all contact forces exerted on the object) seem to be differently affected by single TMS pulses of the motor cortex.
\end{abstract}

(C) 2008 Elsevier Inc. All rights reserved.

Keywords: Grasping; TMS; Motor control

\section{Introduction}

Humans can perform complex manipulative actions with remarkable ease. The flexibility and versatility of use of the human hand is mainly due to its many degrees of freedom, which allow the hand to grasp and manipulate objects in a seemingly infinite number of ways. These observations raise the question of how the motor system is able to control such a highly redundant system, a fundamental problem that occurs both in motor control and robotics $[5,19,49]$. Early studies on the control of the contact forces in the human grasp have focused on the pinch grasp, which involves only the thumb and another finger (review in [27]). More recently, an increasing number of studies has aimed at better understanding how the central nervous system

\footnotetext{
is Part of this work was presented as a preliminary report to First Joint EuroHaptics Conference and Symposium on Haptic Interfaces for Virtual Environment and Teleoperator Systems (WorldHaptics), Pisa, Italy, 18-20 March 2005.

* Corresponding author at: Faculty of Psychology, Vita-Salute San Raffaele University, 58 via Olgettina, 20132 Milan, Italy.

E-mail address: baud-bovy.gabriel@hsr.it (G. Baud-Bovy).
}

controls finger forces in multi-finger grasps, where the problem of motor redundancy is much more acute. As a matter of fact, redundancy is essentially limited to the grip force in the pinch grasp, i.e., to the amount of force used to squeeze an object between two fingers. In contrast, it is possible to vary both the direction and magnitude of the finger forces without violating the task constraints in multi-finger grasps, which involve three or more fingers [52]. To address this problem, it has been hypothesized that the redundant degrees of freedom in the grasp are constrained by prehension synergies, i.e., by conjoint changes of finger forces (reviews in $[43,53]$ ). For example, in the pinch grasp, there is considerable evidence that the grip force is tightly coupled to the load force [27]. In the tripod grasp, a motor synergy constrains the direction of the thumb force toward a point midway between the two opposite fingers even when this solution is sub-optimal for the stability of the grasp [2-4]. Similarly, research on a multi-finger prismatic grasp has individuated two synergies represented by two sets of variables that covary within but not between each set: a grasping synergy that involves a conjoint change of the normal forces and a torque control synergy [47,54]. 
The neuronal correlate of dexterous manipulation is still poorly understood, and experimental evidence often points in different directions. For example, the primary motor cortex (M1) has traditionally been viewed as crucial to a fine and independent control of fingers in humans, which suggests that "upstream" cortical areas are responsible for multi-finger motor synergies. On the other hand, the divergence of the output from single pyramidal neurons and the overlap of the representations of individual fingers in the hand area of M1 suggest that M1 still plays an important role in coordinating finger forces (review in [42]). Single-cell electrophysiological studies in monkeys provide a complex account of the relationship between neuronal activity in M1 and muscular activities. A simple example of this complex relationship is the absence of a fixed relationship between the firing rate of neurons in $\mathrm{M} 1$ and the force in a force production task [24]. Another example is the observation that the activity of some neurons in M1 is related to the intended direction of the force independently of the group of muscles used to produce an action [18,32]. Altogether, these studies suggest that the connectivity between M1 and muscles is not fixed but fluid, and that M1 might in fact code the variable that is the most meaningful to the task rather than the degree of activation of muscles [20]. Both observations run against the idea that fixed and separate assemblies of neurons represent motor synergies in M1. fMRI studies of humans in grasping tasks have shown that the average activity in M1 increases both with the production of large forces and with higher precision or coordination demands, the difference being that more dexterous tasks usually involve a more extended network $[14,15,29]$. As a matter of fact, stronger activity is observed in various bilateral frontal and parietal regions (including ipsilateral M1) during precision grasp than during power grasp [14], as well as during gentle manipulation [29]. For example, the well-known coupling between the load and grip forces in a lift-and-hold task seems to be associated with an area in the right intraparietal cortex [16]. These results support those of electrophysiological studies that attribute an important role to this more extended network for the integration of multimodal sensory information and the selection of a particular grasp $[26,6]$. They do not however provide much information about the possible involvement of these cortical areas in the precise on-line control of finger forces during the manipulation.

In this respect, transcranial magnetic stimulation (TMS) offers a unique opportunity to investigate the role of specific cortical areas in the dexterous manipulation of objects. Indeed, TMS is the only technique that allows one to evaluate the corticospinal pathway functionality in the intact human, from the cortex to the target muscles of the contralateral hemibody [1], and is widely used both in motor control research and in patients with neurological disorders ([36,37] for recent reviews). The technique is based on a brief magnetic pulse which is delivered to the scalp through a coil, and currents induced in the brain may produce excitation or inhibition of superficial cortical neurons [21]: for example, single-pulse TMS applied on the scalp overlying M1 elicits contralateral muscle twitches (called motor evoked potentials, or MEPs), that reflect physiological properties of the corticospinal drive during voluntary $[31,13,44]$ or even imagined [40] motor commands.
Several studies have examined the MEPs during reaching movements that precede the contact with the object. In general, these studies have revealed a precise pattern of cortical activation that is closely related to the muscles required to grasp a given object $[31,7,35]$. A few studies have also examined the effect of TMS on the finger forces in the pinch grasp. The focus in these studies was not on the transient increase of finger forces caused by the muscular twitch but on the longer lasting changes in the finger forces that followed this transient. Stimulation of M1 just before or during contact caused a lengthening of the preload phase and increase of the grip force used while grasping the object during the trial [31]. Short bursts of TMS applied on the ipsilateral M1 150-200 ms before contact lead to an increase of the grip force and to a shortening of the preload phase [12]. These results show the bilateral involvement of M1 in the precise control of grasping forces. Moreover, M1 also seems to play a crucial role in storing previously acquired information about the load since a virtual lesion of M1 induced by repeated TMS disrupted the scaling of the grip forces based on information acquired during a previous lift ([8]; see [34]). Similarly timed TMS of other cortical areas principally disrupted the timing of the lift phase in the case of the dorsal premotor cortex or the positioning of the fingertips in the case of the ventral premotor and parietal cortex $[10,11]$.

Several studies have examined in more detail the characteristics of the transient increase of the finger forces evoked by the TMS of the motor cortex and their relationship with MEPs in single-finger force production tasks (e.g., [25,39,51,33]) or in the pinch grasp $[28,22,23]$. Most of these studies found a context- and muscle-dependent relationship between the level of pre-existing EMG activity and the size of MEP responses to TMS. In addition, the few studies that have investigated the effect of TMS in a multi-finger force production task have revealed a relatively high degree of physiological independence between fingers, since the TMS-evoked force on a finger was dependent on the background force of that finger and independent by forces concurrently produced by the other fingers $[9,30]$. However, forces produced were not finalized into a grasp, which makes the results obtained in these studies hardly comparable with those obtained in the present study.

The goal of the current investigation was to examine the effects of TMS of M1 during the static phase of a three finger grasp. As noted above, motor redundancy is much higher in multi-fingered grasps than in pinch grasps. For this reason, a question of particular interest will be whether the TMS disrupts the finger forces in a manner that also perturbs the control of the net force applied on the object. This study examines the relationship between traditional MEP characteristics and what we have called "motor evoked forces" (MEFs), that is the TMS-evoked variation of the individual finger forces from their baseline level. Besides providing evidence that synergistic MEFs can be measured reliably during the hold phase of a grasp, this work provides preliminary evidence that TMS of M1 affects differently two important characteristics of multi-fingered grasps: the grip force and the net force. 


\section{Subjects and methods}

\subsection{Subjects}

Four right-handed adults participated in the study (one female and three males, age range: $28-45$ ). The subjects gave their written informed consent to the experimental procedures, which was approved by the Local Ethics Committee.

\subsection{Experimental procedure and setup}

Seated participants placed their right arm on a cushioned armrest that allowed the hand to hang freely to provide muscular relaxation between trials. Participants were instructed to grasp the device that measured the finger forces with the thumb, index and middle fingers (Fig. 1A). The device was fixed on the table at a position that allowed one to grasp it with minimal movements from the relaxed hand position. The position of the fingertips was constrained by small vertically oriented contact surfaces (16 mm diameter). Maximum grip force (MGF) was measured at the beginning of the session by instructing the participant to squeeze the object as strongly as possible for a 2-s period.

(A)

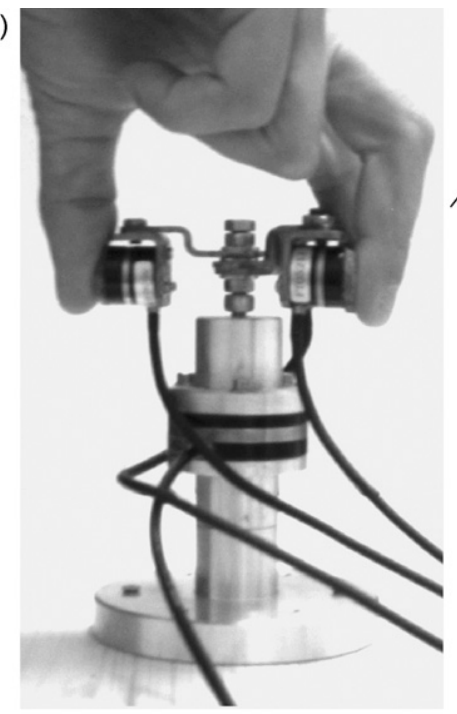

(B)

(C)

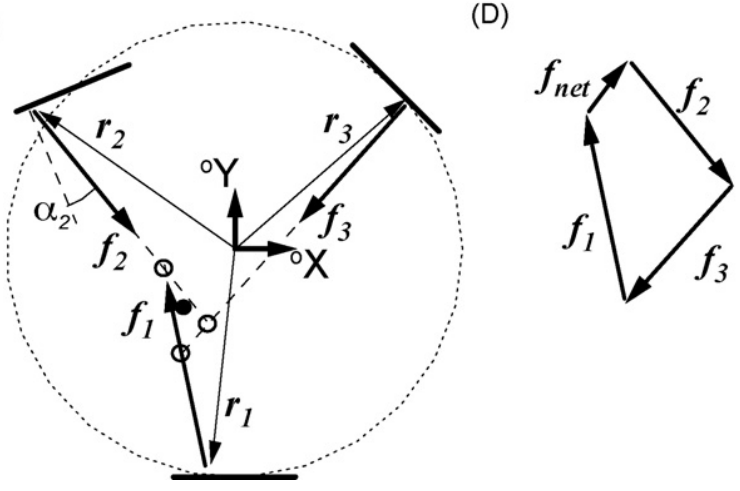

Fig. 1. (A) Experimental device. Three six degrees-of-freedom force/torque sensors are mounted under the contact surface. A fourth one is mounted on the base of the device. (B) The soft-finger model of the contact forces. The finger applies a force $f=\left[f_{x} f_{y} f_{z}\right]$ and a moment $m_{n}$ around the direction of the contact surface normal $n$ at the center of pressure $\left(p_{x} p_{z}\right)$. By definition, the moments around the $x$ and $z$ directions are null at the center pressure. The direction of the contact force must belong to the friction cone to avoid a finger slip. (C) Schematic illustration of the finger forces in the horizontal plan $\left(f_{i}\right)$. The short thick lines represent the contract surfaces. The solid disk represents the force focus, i.e., the average position of the intersections of the three finger forces (empty circle). (D) The force triangle shows how to graphically compute the net force.
The task consisted in maintaining a stable grasp without producing any net force in the horizontal plane. The target level of grip force, defined as the sum of the intensities of all the three forces, corresponded approximately to $7,15,22$ and $33 \%$ of the MGF. Higher forces were not used so as to avoid both muscular and central fatigue mechanisms due to the required effort along task repetitions. Pilot experiments indicated that it was easier to maintain the desired level of grip and net force in the horizontal plane if a force was applied in the vertical direction at the same time. For this reason, participants were also required to apply a small vertical net force (about $10 \%$ of the grip force).

Contact forces were recorded by three small six degrees-of-freedom force/torques (F/T) transducers (ATI Nano-17) mounted under each contact surface. A fourth F/T sensor (ATI Mini 40) was mounted on the shaft that linked the contact surfaces and the table. This sensor was used to monitor the net force exerted by the hand on the instrumented object during the experiment. All the force/torque sensors are connected to the main processing unit via A/D boards (NI PCI 6034E, 16 bits, sampling rate was set to $200 \mathrm{~Hz}$ ). Custom-made software allowed the experimenter to monitor the acquired forces on-line and to save them for off-line analysis.

A monitor provided visual feedback on the grip and net forces. A vertical bar indicated the applied grip force while the position, size and orientation of a square provided information on the applied net force. The position of the square depended on the direction and magnitude of the net force in the horizontal plane, its size depended on the net force in the vertical direction, and its orientation depended on the net torque along the vertical axis. At the beginning of each trial, participants used the visual feedback to bring the grip and net forces to the desired levels. Once both forces had stabilized around their target levels, the experimenter started to record the force data from all four sensors for $2 \mathrm{~s}$. The TMS was automatically triggered after $500 \mathrm{~ms}$. The same trigger signal was used to synchronize force data with the MEPs.

\subsection{Transcranial magnetic stimulation}

TMS was carried out with a Magstim Super Rapid machine (maximal output $2 \mathrm{~T}$ ) connected with an 8 -shaped focal coil. Individual resting excitability thresholds for motor cortex stimulation were first determined by measuring the amplitude of motor twitches evoked by single TMS stimuli in the contralateral first interosseous dorsalis (FDI) muscle. According to International Guidelines [38], motor threshold (MT) was defined as the minimal intensity of the stimulator output capable of evoking a MEP of $>50 \mu \mathrm{V}$ with $50 \%$ probability while the target muscle is at rest. The stimulating coil rested tangential to the scalp surface corresponding to the primary motor cortex, with its handle pointing backwards and angled at about $45^{\circ}$ from the midline. Such position was marked on a strictly adherent bathing cap, so that coil replacement across experimental sessions was accurate. Four different stimulation intensities corresponding to 100, 111, 122 and $133 \%$ of the individual MT level were applied for each of the four initial force grip condition yielding a total of 16 experimental conditions. Trials inside each condition were divided into four blocks of three trials yielding a total of 12 repetitions per condition and 192 trials per participant. The order of presentation of the blocks was randomized.

\subsection{Motor evoked potentials (MEPs)}

The MEPs from three contralateral muscles involved in the grasping were recorded in a standard belly-tendon montage (disposable surface electrodes) for the first subject: right extensor communis digitorum (ECD), the flexor communis digitorum (FCD) and the first interosseous dorsalis muscles. The MEPs from a fourth muscle, the abductor pollicis (AbP) were recorded for the other subjects. Electromyographic signals (from -45 to $155 \mathrm{~ms}$ around the TMS instant) were filtered (20-2 kHz), sampled at $4 \mathrm{kHz}$, and stored for off-line analysis. The EMG signals of the first three subjects were subsequently examined visually to identify the latency, peak-to-peak amplitude and area. We discarded a total of 20 trials (about 10\%) of one participant for technical reasons and book-keeping problems during the last experimental session prevented the analysis of the MEPs of the last subject. Trials with missing MEP were excluded from further analysis. 


\subsection{Finger force analysis}

In the tripod grasp, the object is grasped by the thumb, index and middle fingers. The mechanical interaction taking place between each finger and the object was modeled by a force $f_{i}=\left[f_{x} f_{y} f_{z}\right]$ applied at the center of pressure $r_{i}$ and a torque $m_{i}=\left[0 m_{n} 0\right]$, about the normal $n$ passing through the center of pressure and perpendicular to the surface of the object (Fig. 1B). This kind of interaction at the contact point has been studied in robotics and the corresponding model is referred to as the soft-finger contact model [41].

The contact forces must satisfy the squeezing and frictional constraints to insure the stability of the grasp. The squeezing constraint

$n^{T} f \geq 0$

states that a finger can only push and not pull the contact surface. The frictional constraint

$\left\|\left(\mathrm{I}-n n^{T}\right) f\right\|=\mu f^{T} n$

states that the contact force must belong to the friction cone to avoid a finger slip. The aperture angle of the friction cone is $\tan ^{-1}(\mu)$ where $\mu$ is the linear coefficient of friction. The normal torque $m_{n}$ should also satisfy a similar frictional constraint

$m_{n} \leq \mu_{\text {rot }} f^{T} n$

where $\mu_{\text {rot }}$ is the rotational coefficient of friction.

In addition, the equilibrium equations state the relation between the contact forces and the net force:

${ }^{0} f_{\text {net }}=\sum_{i}^{0} f_{i}$

${ }^{0} m_{\text {net }}=\sum_{i}^{i} r_{i} \times{ }^{0} f_{i}+{ }^{0} m_{i}$

where ${ }^{0} f_{i}$ and ${ }^{0} m_{i}$ are the contact force and moments of the $i$ th digit expressed in the object coordinate system $\left[{ }^{0} X,{ }^{0} Y,{ }^{0} Z\right]$, and $r_{i}=\left[r_{x i} r_{y i} r_{z i}\right]$ is the position of the center of pressure relative to center of mass (Fig. 1C). When the net force is null in the tripod grasp, the force directions intersect at a point known as the force focus [52]. When the net force was not null, we used the mean position of the three pair-wise intersection points of the three finger force directions (Fig. 1C).

For each finger, we computed the norm of the contact force in the horizontal plane

$f_{\mathrm{hi}}(t)=\sqrt{f_{x i}^{2}(t)+f_{y i}^{2}(t)}$

where the index $i$ identifies the finger (thumb, index and middle fingers). The grip force refers to the sum of the magnitude of the three contact forces:

$f_{\text {grip }}(t)=\sum_{i=1}^{3} f_{\mathrm{hi}}(t)$.

Intuitively, the grip force represents the level of squeezing used by the participant to grasp the object. The net force refers to the magnitude of the linear component of the net force $f_{\text {net }}$ force in the same plane:

$f_{\text {net }}(t)=\sqrt{f_{\text {net }_{x}}^{2}(t)+f_{\text {net }_{y}}^{2}(t)}$.

In this article, we use the term motor evoked force to refer to the shor variation of the contact force above or below the stable level of force reached when the stimulation is evoked by TMS of M1. For each trial, we measured the characteristics of the MEF for each one of the three contact forces $f_{\mathrm{hi}}$, as well as for the grip force $f_{\text {grip }}$ and net force $f_{\text {net }}$ by identifying the latency, peak time, size and directional shift of the first peak that followed the stimulation. Latency was defined as the first instance when the force exceeded 3.5 standard deviation of a $100 \mathrm{~ms}$ window. The MEF size and angle correspond to the difference in the force magnitude and direction between the time of the stimulation and peak time. A well-defined MEF is a MEF that has a latency of less than $50 \mathrm{~ms}$, a peak time of less than $120 \mathrm{~ms}$, and amplitude of at least $0.15 \mathrm{~N}(\sim 15 \mathrm{~g})$. We substituted the missing values for the MEF sizes with the threshold value $(0.15 \mathrm{~N})$ in all trials where an effect of the stimulation could be seen in at least one finger or the grip force, in order not to overestimate the average MEF size by excluding trials where the TMS was demonstrably effective but had an effect too small to be detected on all traces.

\section{Results}

\subsection{Grip and net forces before TMS}

The grip force is the sum of the three finger forces and represents the total amount of force with which subjects squeezed the object. The mean grip force \pm S.D. at stimulation time was $7.6 \pm 1.0,14.5 \pm 1.4,21.8 \pm 1.1$ and $32.1 \pm 1.3 \%$ of MGF, which closely matched the desired levels of initial grip force (7, 15, 22 and 33\% of MGF, respectively). The net force at stimulation time ranged from $0.6 \pm 0.4$ to $1.6 \pm 1.1 \mathrm{~N}$ depending on the subject, which corresponds to $3 \pm 2$ and $13 \pm 8 \%$ of the grip force at stimulation time (see $f_{\mathrm{h}}^{\text {net }}$ and $f_{\mathrm{h}}^{\text {net }} / f_{\mathrm{h}}^{\text {grip }}$ in Table 1 ). The deviations from the desired absence of net force in the horizontal plane were the largest for the second participant, and increased with the initial level of grip force (see insets in Fig. 2).

\subsection{Finger forces before TMS}

As noted in the introduction, the magnitude and direction of the finger forces are only partially constrained by the equilibrium conditions in the tripod grasp. Fig. 2 shows the average finger forces and position of the force focus - point of intersection between the three forces - for each subject and grip force level just before the stimulation. Table 1 reports the magnitude of each finger force expressed as percentage of the grip
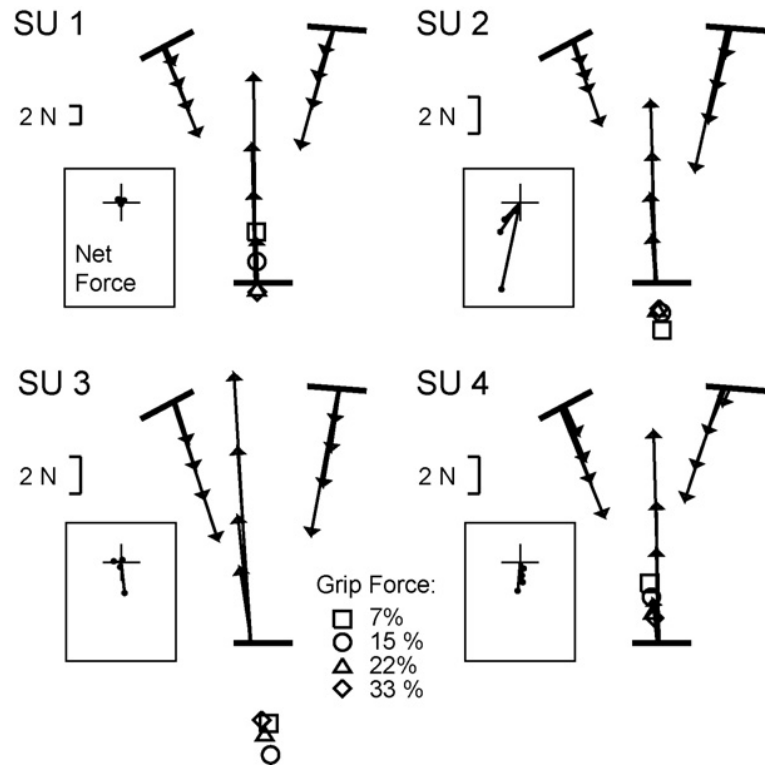

Fig. 2. Finger forces in the horizontal plane before TMS. Arrows denote the average finger forces for each subject and initial level of grip forces. The symbols corresponding force focuses, i.e., the points of intersection of the three forces directions (square: $7 \%$ of MGF, circle: $15 \%$, triangle: $22 \%$, inverted triangle: $33 \%)$. Insets: Net force in the horizontal plane for each level of grip force. 
Table 1

Main characteristics of the finger forces, MEFs and MEPs

\begin{tabular}{|c|c|c|c|c|c|}
\hline \multirow{4}{*}{$\begin{array}{l}\text { Sex } \\
\text { MGF (N) }\end{array}$} & \multicolumn{4}{|l|}{ Subject } & \multirow[t]{2}{*}{ Mean } \\
\hline & 1 & 2 & 3 & 4 & \\
\hline & M & $\mathrm{F}$ & M & M & - \\
\hline & 150 & 70 & 98 & 76 & - \\
\hline \multicolumn{6}{|c|}{ Finger forces before stimulation } \\
\hline$f^{\text {net }}(\mathrm{N})$ & $0.6 \pm 0.4$ & $1.6 \pm 1.1$ & $0.9 \pm 0.8$ & $0.7 \pm 0.5$ & $1.0 \pm 0.7$ \\
\hline$f^{\text {net }} / f^{\text {grip }}(\%)$ & $3 \pm 2$ & $13 \pm 8$ & $5 \pm 4$ & $7 \pm 5$ & $7 \pm 5$ \\
\hline$f^{\text {thumb } / f^{\text {grip }}(\%)}$ & $49 \pm 1$ & $47 \pm 6$ & $49 \pm 3$ & $47 \pm 3$ & $48 \pm 3$ \\
\hline$f^{\text {index }} / f^{\text {grip }}(\%)$ & $23 \pm 3$ & $21 \pm 5$ & $27 \pm 3$ & $29 \pm 5$ & $25 \pm 4$ \\
\hline$f^{\text {middle }} / f^{\text {grip }}(\%)$ & $29 \pm 3$ & $33 \pm 5$ & $25 \pm 3$ & $25 \pm 4$ & $28 \pm 4$ \\
\hline$\alpha^{\text {thumb }}$ (degree) & $-1.2 \pm 3.3$ & $-2.9 \pm 3.5$ & $-5.3 \pm 3.2$ & $-2.2 \pm 3.6$ & $-2.9 \pm 3.4$ \\
\hline$\alpha^{\text {index }}($ degree $)$ & $4.4 \pm 5.5$ & $9.7 \pm 4.8$ & $10.3 \pm 3.0$ & $5.1 \pm 4.0$ & $7.4 \pm 4.3$ \\
\hline$\alpha^{\text {middle }}$ (degree) & $12.0 \pm 4.2$ & $8.3 \pm 4.4$ & $5.0 \pm 3.6$ & $16.3 \pm 5.0$ & $10.4 \pm 4.3$ \\
\hline \multicolumn{6}{|c|}{ Percentage of trials with well-identified MEF } \\
\hline \# Thumb (\% trials) & 78.1 & 92.7 & 92.2 & 81.8 & 86.2 \\
\hline \# Index (\% trials) & 71.4 & 94.3 & 96.4 & 73.4 & 83.9 \\
\hline \# Middle (\% trials) & 72.4 & 96.9 & 90.1 & 81.2 & 85.2 \\
\hline \# Grip (\% trials) & 82.8 & 97.9 & 97.4 & 86.5 & 91.1 \\
\hline \# Net (\% trials) & 33.3 & 79.7 & 61.5 & 50.5 & 56.3 \\
\hline \multicolumn{6}{|l|}{ Motor evoked forces } \\
\hline$\Delta f^{\text {net }} / f^{\text {grip }}(\%)$ & $3 \pm 3$ & $21 \pm 15$ & $12 \pm 9$ & $13 \pm 12$ & $12 \pm 10$ \\
\hline$\Delta f^{\text {thumb }} / f^{\text {thumb }}(\%)$ & $20 \pm 13$ & $69 \pm 47$ & $42 \pm 31$ & $55 \pm 42$ & $47 \pm 33$ \\
\hline$\Delta f^{\text {index }} / f^{\text {index }}(\%)$ & $22 \pm 16$ & $63 \pm 38$ & $50 \pm 39$ & $43 \pm 33$ & $45 \pm 31$ \\
\hline$\Delta f^{\text {middle }} / f^{\text {middle }}(\%)$ & $22 \pm 17$ & $89 \pm 49$ & $67 \pm 53$ & $76 \pm 68$ & $64 \pm 47$ \\
\hline$\Delta f^{\text {thumb }} / \Delta f^{\text {grip }}(\%)$ & $50 \pm 9$ & $45 \pm 16$ & $41 \pm 8$ & $49 \pm 10$ & $46 \pm 11$ \\
\hline$\Delta f^{\text {index }} / \Delta f^{\text {grip }}(\%)$ & $22 \pm 5$ & $20 \pm 6$ & $27 \pm 5$ & $21 \pm 7$ & $22 \pm 6$ \\
\hline$\Delta f^{\text {middle }} / \Delta f^{\text {grip }}(\%)$ & $30 \pm 11$ & $43 \pm 7$ & $33 \pm 8$ & $36 \pm 12$ & $35 \pm 10$ \\
\hline$\Delta \alpha^{\text {thumb }}(\operatorname{deg})$ & $0.2 \pm 0.6$ & $2.7 \pm 2.5$ & $1.3 \pm 2.2$ & $-1.3 \pm 1.9$ & $0.7 \pm 1.8$ \\
\hline$\Delta \alpha^{\text {index }}(\operatorname{deg})$ & $2.0 \pm 2.1$ & $-0.6 \pm 2.2$ & $4.5 \pm 3.1$ & $3.7 \pm 3.1$ & $2.4 \pm 2.6$ \\
\hline$\Delta \alpha^{\text {middle }}(\mathrm{deg})$ & $-1.8 \pm 1.9$ & $-2.1 \pm 2.0$ & $0.5 \pm 2.4$ & $-8.1 \pm 4.7$ & $-2.9 \pm 2.7$ \\
\hline \multicolumn{6}{|l|}{ Motor evoked forces } \\
\hline \# ECD (\% trials) & 97.4 & 98.3 & 98.4 & - & 98.0 \\
\hline \# FCD (\% trials) & 98.4 & 98.3 & 93.8 & - & 96.8 \\
\hline \# FDI (\% trials) & 100 & 98.3 & 100 & - & 99.5 \\
\hline \# AbP (\% trials) & - & 98.3 & 95.3 & - & 96.7 \\
\hline ECD latency (ms) & $14.4 \pm 0.6$ & $18.2 \pm 0.5$ & $18.2 \pm 0.7$ & - & $16.9 \pm 0.6$ \\
\hline FCD latency (ms) & $14.6 \pm 0.5$ & $18.4 \pm 0.6$ & $19.5 \pm 0.8$ & - & $17.5 \pm 0.7$ \\
\hline AbP latency (ms) & $20.4 \pm 0.7$ & $22.6 \pm 0.9$ & $23.3 \pm 0.9$ & - & $22.1 \pm 0.8$ \\
\hline AbP latency (ms) & - & $23.7 \pm 1.0$ & $24.0 \pm 0.7$ & - & $23.8 \pm 0.9$ \\
\hline
\end{tabular}

force $\left(f_{\mathrm{h}}^{\text {finger }} / f_{\mathrm{h}}^{\text {grip }}\right)$ and the mean angle ( $\left.\alpha^{\text {finger }}\right)$ between the finger force and the contact surface normal for each subject. For all participants, the thumb force was about half of the grip force $\left(48 \pm 3 \%\right.$, see $f_{\mathrm{h}}^{\text {thumb }} / f_{\mathrm{h}}^{\text {grip }}$ in Table 1$)$. The second largest force could be the index or middle finger force depending on the participant. The initial grip force did not markedly affect the distribution of the force across the fingers. In fact, the contribution of each finger to the grip force varied by about $4 \%$ in the experiment (see S.D.s of $f_{\mathrm{h}}^{\text {finger }} / f_{\mathrm{h}}^{\text {grip }}$ in Table 1). For all participants, the thumb force was directed toward a point midway between the index and middle fingers. There were small differences in the direction of the index and middle finger forces across participants, but they pointed in general toward the thumb (the force focuses are grouped in the vicinity of the thumb position, see Fig. 2). The initial direction of the finger forces varied little in the experiment (average S.D. of $\alpha^{\text {finger }}$ was about $4^{\circ}$, see Table 1 ). Moreover, the initial level of grip force did not affect markedly the initial direction of the finger forces (note that the force focuses corresponding to different levels of initial grip forces in Fig. 2 are grouped together).

\subsection{Presence/absence of MEPs and MEFs}

Fig. 3A shows the magnitude of the finger and net forces in the horizontal plane $\left(f_{\mathrm{h}}\right)$ during a trial where the TMS (133\% of MT) caused a well-defined MEP on all recorded EMGs. Note that the main pulse of the MEPs is extremely brief ( $\sim 25 \mathrm{~ms})$ and is followed by a period of relative inactivity (cortical silent period) that lasts much longer (up to $200 \mathrm{~ms}$ depending on the muscle and background activity). The muscle contractions caused a transient increase of the finger forces (or MEF) that lasted about 100-150 ms. In this particular trial, the net force did not vary markedly, which indicates that the increase of finger forces remained balanced throughout the trial. 
(A)
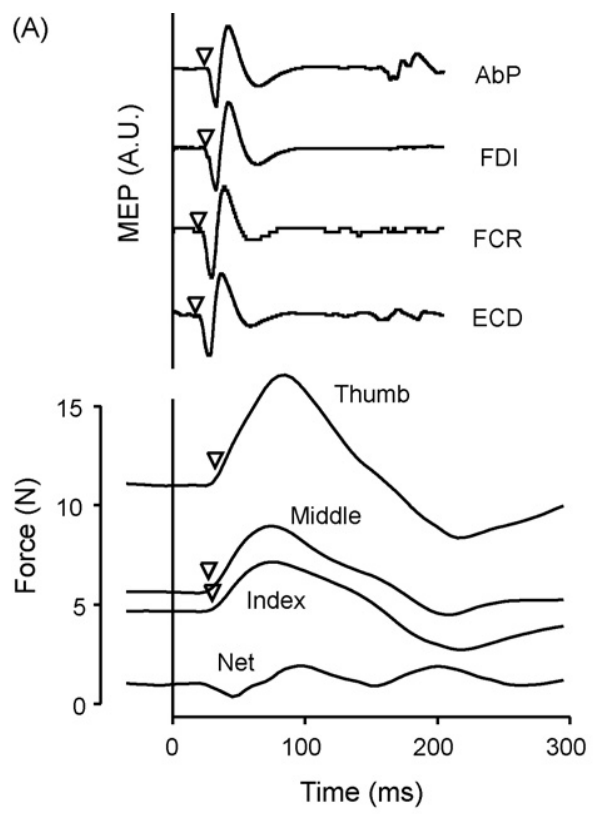

(B)

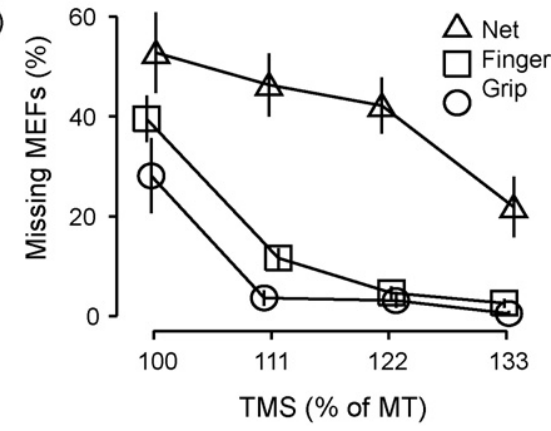

Fig. 3. (A) MEPs and MEFs during sample trial (SU 4, initial grip force: $22 \%$ of MGF, TMS: $133 \%$ of MT). Triangles denote the latencies of the corresponding MEP or MEF. Note the absence of a well-defined MEF on the net force in spite of marked increases of the finger forces. (B) Mean percentage of missing MEFs for the finger forces (square), grip force (circle) and net force (triangle). Percentages for thumb, index and middle fingers have been pooled because they were similar. Vertical bars represent the standard error $(N=16$ for grip and net force and $N=48$ for finger forces).

TMS caused a MEP in $98 \%$ of the trials (all subjects and muscles pooled together, see Table 1). The missing MEPs occurred at various TMS intensities and initial grip force levels and were probably caused by tiny variations in the positioning of the coil and/or the state of the subject. Interestingly, a MEP was almost always present at the lowest TMS intensity (100\% of MT) and initial grip force level (7\% of MGF). Only about $3 \%$ of the MEPs were missing in this condition.

The TMS caused a well-identified MEF in at least one of the finger force or the grip force in $92 \%$ of the trials (all subjects pooled, $N=768$ ). This percentage ranged from 85 to $99 \%$ depending on the subject (see Table 1). Almost all trials with a MEF in at least one of three finger forces had also valid MEF in the grip force and vice-versa. In $84 \%$ of these trials, a MEF could be observed in all three finger forces. In contrast to the percentages associated with the finger or grip forces, the percentage of trials where the TMS caused a well-identified MEF in the net force was much lower (only $56 \%$ of the total number of trials, all subjects pooled). This percentage ranged from 33 to $80 \%$ depending on the subject (see Table 1). This lower percentage is due to the fact that the variations of the finger forces often cancelled each other out and did not cause any variation of the net force. In fact, a MEF was observed in one of the finger forces or in the grip force in $84 \%$ of the trials without a MEF in the net force, indicating that the stimulation was effective in most of these trials. In other words, the TMS caused an observable temporary increase of the grip force or one of the finger forces without a noticeable effect on the net force in $36 \%$ of the total number of trials. This observation reveals that in more than a third of the trials, the effect of the stimulation on the finger forces was balanced. The percentage of trials with a well-defined MEF depended mostly on the intensity of the TMS (Fig. 3B). Most of the missing MEFs occurred at the smallest TMS level for the finger forces and the grip force. For the net force, the percentage of well-defined MEFs increased linearly with the TMS level from 47 to reach $78 \%$ at the highest TMS level. The difference between the number of well-defined MEFs of the grip and net forces was largest at the two intermediary TMS level: at these levels, 41 and $39 \%$ of trials did not exhibit any MEF in the net force even though a MEF could be seen in the grip force.

\subsection{Effect of the stimulation on the finger forces}

For all trials and traces where a MEF could be identified, we computed the MEF size (i.e., the difference between the amplitudes of a finger force at stimulation and peak times) and the MEF angle (i.e., the angular difference of a finger force between stimulation and peak times). The TMS had a more marked effect on the amplitude than on the direction of the finger forces. The MEF size ranged from 20 to $90 \%$ of the corresponding finger force at the stimulation time, depending on the finger and subject (see $\Delta f^{\text {finger }} / f^{\text {inger }}$ in Table 1). In contrast, the changes in the direction of the finger forces were in general quite small, in particular for the thumb (average value \pm S.D. for the thumb: $0.7 \pm 1.8^{\circ}$, see $\Delta \alpha^{\text {finger }}$ in Table 1$)$. The increase of a finger force caused by the TMS ( $\Delta f^{\text {finger }}$ ) relative to the increase of the grip force $\left(\Delta f^{\text {grip }}\right)$ was similar to the ratio of the initial finger force (finger) over the grip force ( $f^{\text {grip }}$ ): $46 \pm 11$ vs. $48 \pm 3$ for the thumb, $22 \pm 6$ vs. $25 \pm 4$ for the index finger, and $35 \pm 10$ vs. $28 \pm 4$ for the middle finger $\left(\Delta f^{\text {finger }} / \Delta f^{\text {grip }}\right.$ vs. $f^{\text {finger }} / f^{\text {grip }}$, see Table 1). This shows that the distribution of the grip force across fingers was identical whether one considers the voluntary forces before the stimulation or the involuntary amplitude changes resulting from the TMS. Note however that the standard deviations for the MEF ratios were considerably higher, which reflects the fact that the distribution of the effect of the TMS across finger varied from trial to trial.

To investigate the evolution of the MEFs in time, we computed the latency and peak time of all MEFs for each trial. Fig. 4A represents the average MEF size during a $400 \mathrm{~ms}$ period following the stimulation (all subjects and experimental condition pooled together). These MEFs represent the change in magnitude of the finger $\left(f_{\mathrm{h} i}\right)$, grip $\left(f_{\text {grip }}\right)$ and net $\left(f_{\text {net }}\right)$ forces in the horizontal plane caused by the stimulation. The gross features of 
(A)

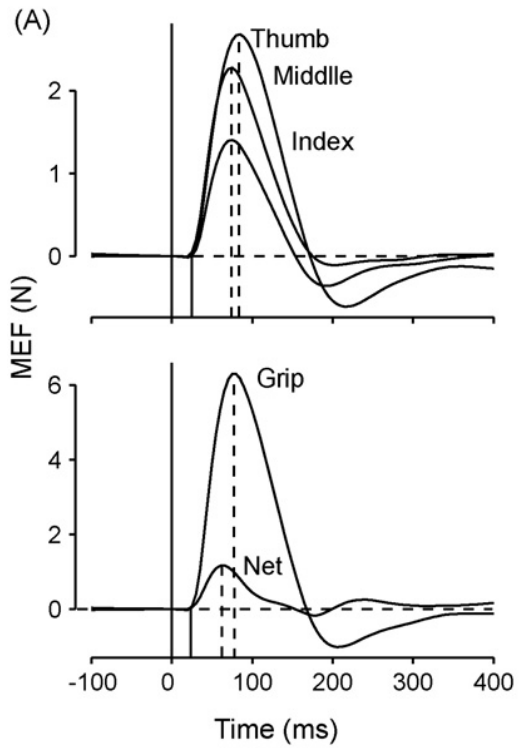

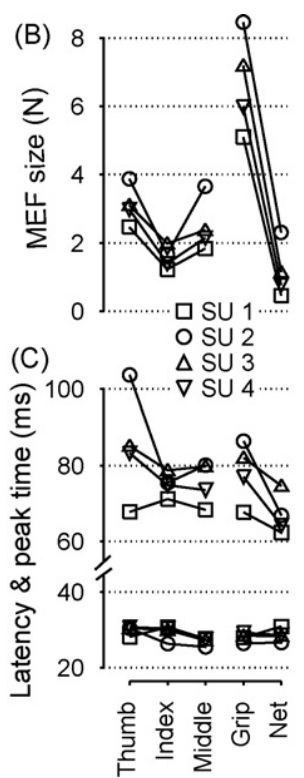

Fig. 4. (A) Average MEFs. The top panel represents the average finger $\operatorname{MEF}\left(f_{\mathrm{h} i}\right)$ while the bottom panel represent the average grip $\left(f_{\text {grip }}\right)$ and net $\left(f_{\text {net }}\right)$ MEF. All repetitions, experimental conditions and subjects are pooled $(N=768)$. The time origin $t=0$ corresponds to the time of the TMS. Vertical dashed lines represent the latencies and peak time of the plotted time series. To show better the effect of the stimulation, we aligned individual time series to their base level (horizontal line) by subtracting to each time series the force level reached at stimulation time. (B) Average MEF size for the thumb, index and middle fingers, grip and net forces across all experimental conditions. Empty squares, circles, triangles and inverted triangles refer to the averages of the four subjects. (C) Average latencies and peak times.

the MEFs were similar across subjects. All MEFs had a latency of about $30 \mathrm{~ms}$, and corresponded to a relatively brief ( $\sim 150 \mathrm{~ms})$ increase of the squeezing force with which the subjects grasped the object, that peaked around $75-80 \mathrm{~ms}$ after the stimulation, followed by a $50-100 \mathrm{~ms}$ long depression below the base level (i.e., the level of force at the time of the stimulation). Then, depending on the subject, the finger forces could either reach again their base level or not. For all subjects, the MEF size was largest for the thumb, followed by the middle and index fingers (Fig. 4B). However, when the MEF size was reported to the magnitude of the corresponding finger force before stimulation, the thumb and index finger forces increased on average only by about $46 \%$ while the middle finger force increased by $64 \%$ (see $\Delta f^{\text {finger }} / f^{\text {finger }}$ Table 1 ). In other words, this observation suggests that thumb and index finger were proportionally the least affected fingers by the TMS.

The latencies for the MEFs did not differ much across fingers or participants (Fig. 4C). In contrast, there were much more marked difference in the peak times for the different MEFs. For all but one subject, the index and middle finger MEF peaked about $11 \mathrm{~ms}$ before the thumb $\operatorname{MEF}(t(588)=20.85$, $P<0.001$; paired $t$-test of the difference between index and thumb MEF peak times for all trials for which peak times could be identified, all subjects and experimental condition pooled; $t(588)=22.24, P<0.001$, same test for the difference between middle finger and thumb). For all subjects, the net force peaked before the grip force or any of the individual finger forces. The peak time difference $(13 \mathrm{~ms})$ between the grip and net force was highly statistically significant $(t(429)=20.5, P<0.001$, paired $t$-test with data from all subjects and conditions pooled together).

To examine whether the TMS caused synergistic changes in the finger forces, we computed the correlation coefficients between the MEF sizes and MEF angles for each pair of fingers (pooling all experimental conditions together). The changes in the magnitudes of the three finger forces were highly correlated among themselves ( $r$ from 0.79 to 0.86 ) and with the grip force ( $r$ from 0.90 to 0.95 ). The effect of the TMS on the magnitude of the net force was best correlated with MEF size of the individual fingers and of the grip force ( $r$ from 0.52 to 0.81 ). In contrast, MEF sizes were only weakly correlated with changes in the direction of the forces ( $r$ from -0.25 to 0.24 ) and these directional shifts were also weakly correlated among themselves ( $r$ from -0.04 to 0.37 , see Fig. 5A). This pattern was present in all subjects. This analysis shows that the distribution of the grip force across the three fingers remained constant not only before the stimulation, when different grip forces were used to grasp the object, but also during the passive movement induced by the TMS pulse. In other words, the effect of TMS can be described at least as a first approximation as a proportional increase of the finger forces. To verify this hypothesis, we computed the correlation between the magnitude of the finger forces before the TMS and the MEF size. This analysis was conducted separately for each level of TMS to isolate the effect of the initial grip force on the MEF size. Fig. 5B shows that the expected pattern was more obvious with strong stimulations. At lower stimulation intensities, the coefficients of correlations were generally not statistically significant.

\subsection{Effect of initial grip force and TMS intensity on the MEFs}

The TMS varied from 100 to $133 \%$ of the MT while the initial grip force level from 7 to $33 \%$ of the MGF. In this section, we examine the effect of these experimental factors on the amplitude, direction, latency and peak time of the finger forces. The effect of initial grip force and TMS intensity on the MEF sizes was complex. For the two first subjects, the effect of the stimulation on the finger and grip forces depended on both factors $(P<0.001$, two-way ANOVAs). Only the stimulation level markedly affected the finger and grip forces of the third participant while the last subject exhibited an interaction pattern, revealing that the initial grip force affected the MEFs only at the strongest TMS (Fig. 6A). Only the grip MEF size is represented in Fig. 6A because the grip force was highly correlated with the individual finger forces (see later) and presented therefore similar characteristics, differing only by a scaling factor. With respect to the net force (Fig. 6B), we found that it showed a marked increase only for the highest level of TMS for three out of the four subjects. Individual two-way ANOVAs of the net force without these trials revealed that the size of the net MEF did not depend on either factor for the first and fourth subjects $(P>0.05)$. For the third subject, the main effect of TMS level was still statistically significant $(P<0.01)$. A slightly different 
(A)

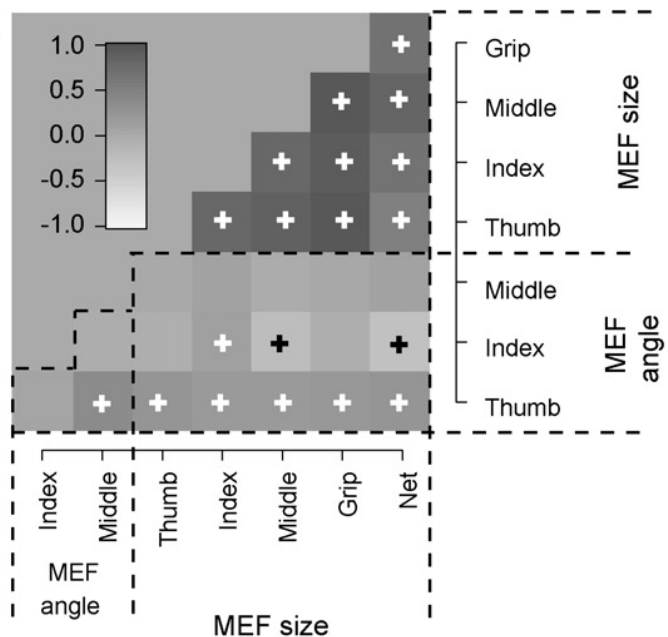

(B)

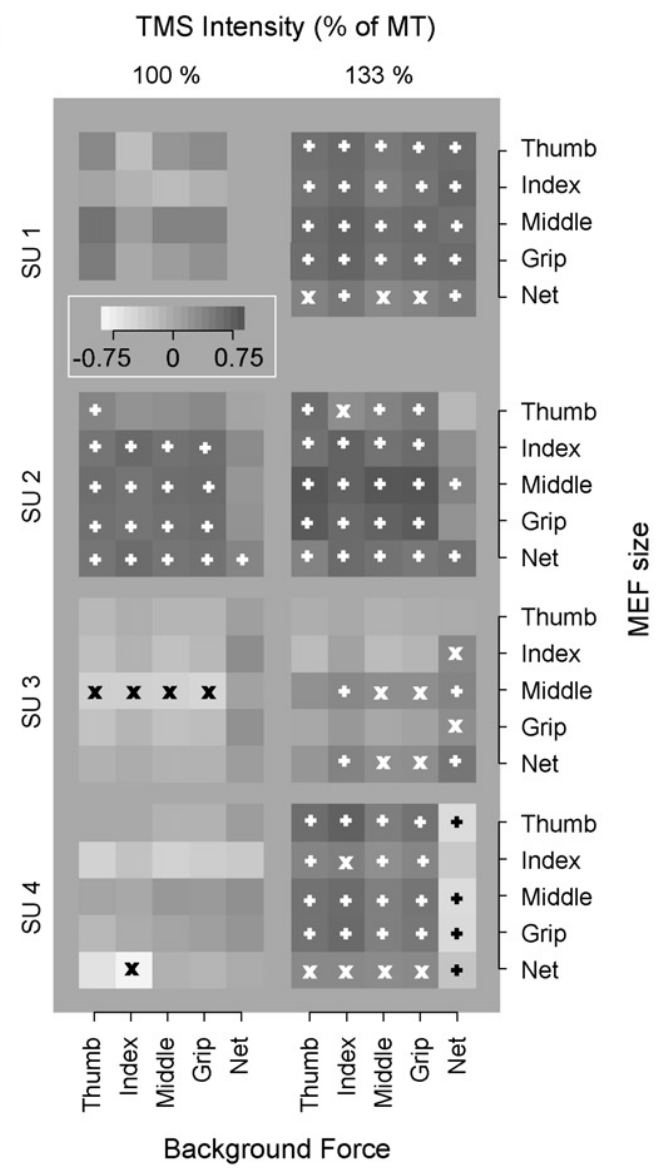

Fig. 5. (A) Correlation between MEF size and MEF angles (all subjects and experimental condition pooled together). The + and $\times$ signs denote that the coefficient of correlation was statistically significant at the level 0.01 and 0.05 , respectively. (B) Correlation between the MEF sizes and the background forces (measured at the time of the stimulation, $t=0$ ). The coefficients of correlations were computed for each subject and level of intensity separately (only the lowest and highest stimulation levels are represented).

view emerged when the MEF sizes were expressed in percents of the initial grip force (Fig. 6C). In this case, the MEF sizes still increased with the TMS intensity, but they decreased with the level of initial grip force, showing that the effect of the stimulation was proportionally more contained when the participants

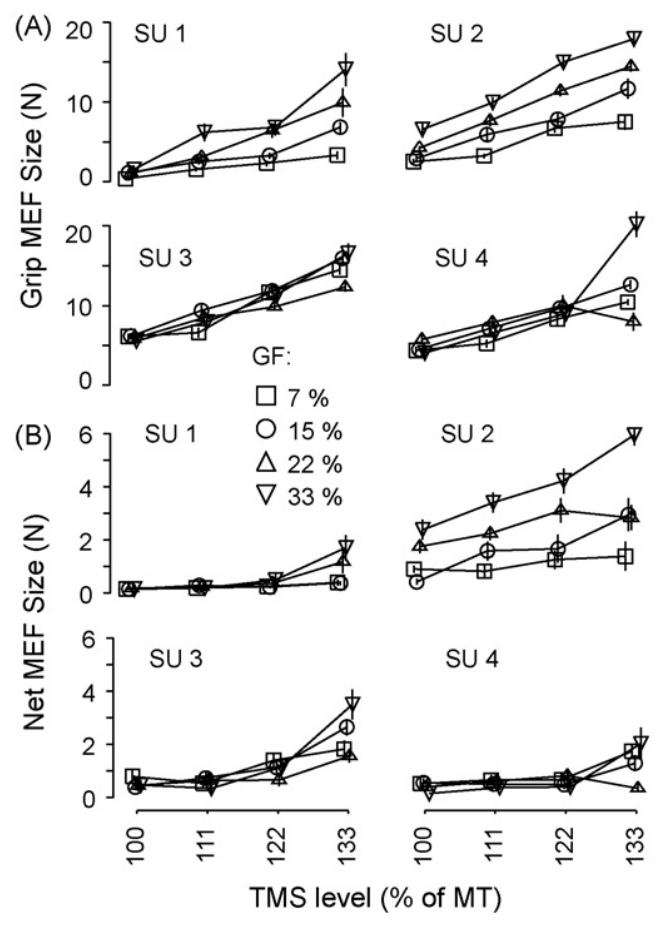

(C)
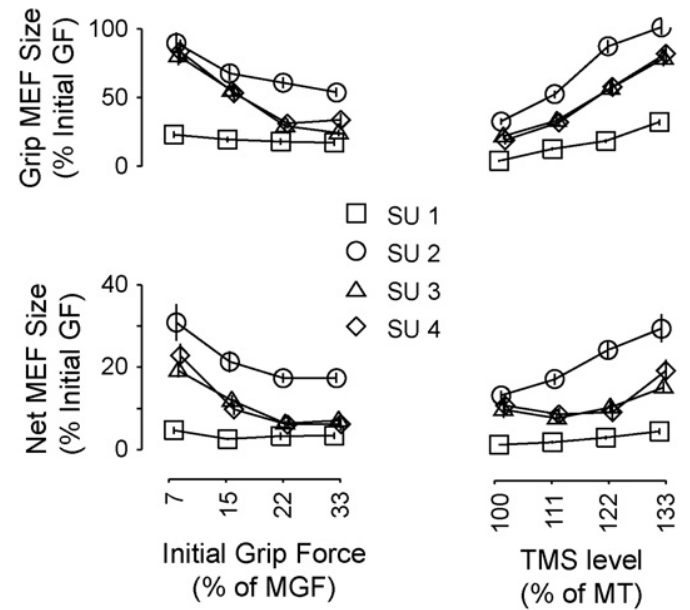

Fig. 6. (A and B) Average MEF size for the grip and net force as a function of the TMS intensity and initial level of grip force. Each panel plots the data of a different subject. The symbols denote various levels of grip force $(7,15$, 24 and $33 \%$ of maximum grip force, respectively). Vertical bars represented the standard error $(N=16)$. (C) Normalized MEF size (expressed as a percentage of the initial grip force) as a function of the initial grip force level (left) or of the TMS intensity (right). The symbols denote the average for each subject.

squeezed the object more. These observations held true for all fingers as well as the grip and net forces.

The initial grip force had a more marked effect on the direction of the finger forces than the grip forces. While both main effects were in general statistically significant, the $F$ values associated with the initial grip force were in general much larger than $F$ values associated with TMS (the inverse was true only for the middle finger of the first subject, one of the fifteen [three fingers by five participants] two-way ANOVAs of the MEF angles conducted). In general, interactions were not statistically significant. MEF angles tended to decrease when the initial grip force 


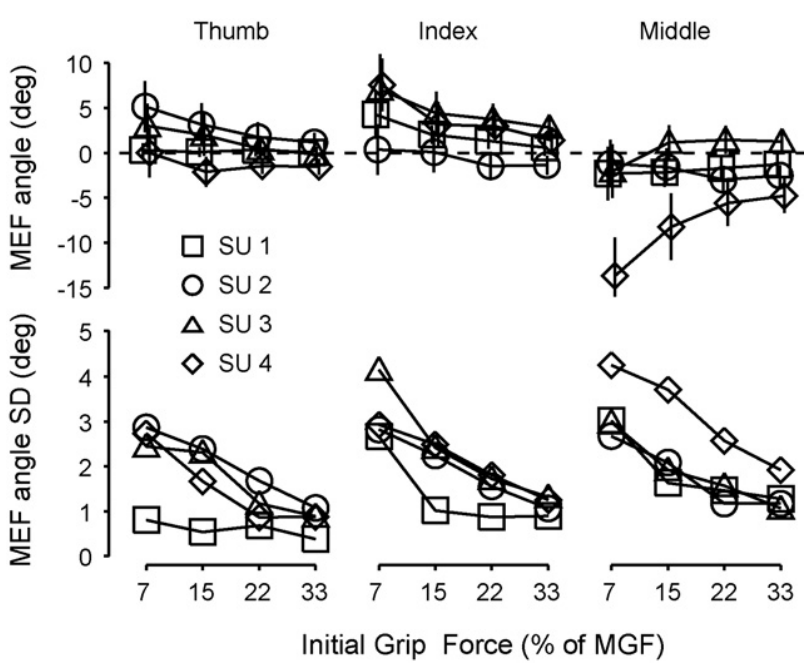

Fig. 7. Systematic (top) and random (bottom) effects of the TMS on finger force directions (MEF angles) as a function of the initial level grip force. The symbols denote average value of for each subject. The vertical bars in the top panels represent the standard deviation of the MEF angles and correspond to the standard deviations plotted in the bottom panels.

increased (top row of Fig. 7), indicating that the effect of the TMS on the force direction was reduced when the object was grasped more strongly. The difference is particularly noticeable for the middle finger of the last participant (SU 4), where the directional shift more than halved with a stronger grasp. It is also noteworthy that the standard deviation of the MEF angles decreased with the initial grip force (see bottom row of Fig. 7), indicating that the directional shift caused by the stimulation became not only smaller but also less variable when the object was grasped more strongly.

The results for the MEF latencies and peak times were simpler. For all subjects, the initial grip force level and the TMS did not affect the latencies (in the two-way ANOVAs of the latencies, only one out of 45 [five participants by three fingers by three possible effects] tests was statistically significant, $P<0.001=0.05 / 45$, Bonferonni correction). In any case, Fig. 8 shows that the latencies did not vary, by more than a few milliseconds, if at all. In contrast, we found a very large effect of the initial grip force on the peak times of all MEFs, which

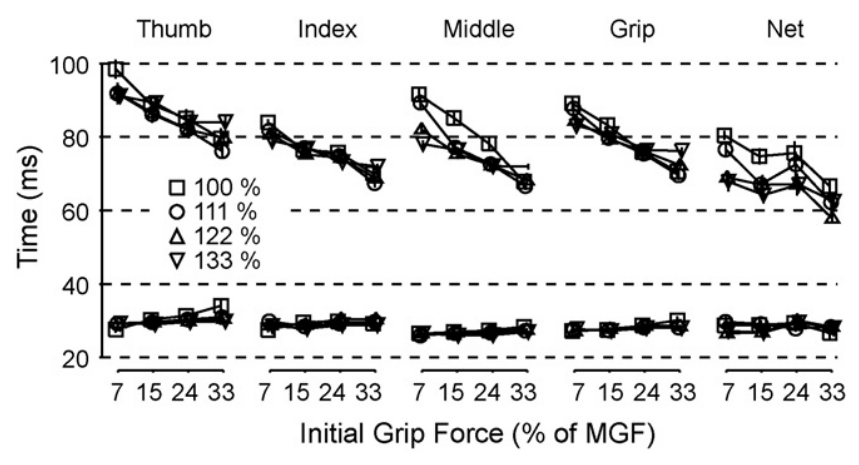

Fig. 8. Average MEF latencies and peak times as a function of the initial levels of grip force and TMS intensities (data from all subjects are pooled together). The symbols denote various levels of grip forces $(7,15,24$ and $33 \%$ of maximum grip force, respectively). Vertical bars represent the standard error $(N=64)$. decreased by about $18 \mathrm{~ms}$ (or 20-25\%) between the most gentle and strongest grasp (Fig. 8). We also found a statistically significant effect of TMS intensity that corresponded to a decrease of peak times for stronger stimulations but this effect was much weaker. As a matter of fact, the $F$ values associated with the initial grip force effect were at least twice as large as the $F$ values associated with the TMS intensity effect for 17 out of the 20 [four MEFs by five participants] two-way ANOVAs performed and, in the remaining three cases, the size of the two effects was approximately equal. For the net force, the size of the two effects was equal for the first three participants, and only the initial grip force was statistically significant for the last participant.

\subsection{Motor evoked potentials (MEPs)}

We computed the coefficients of correlation between the amplitude and area for each muscle and subject separately, pooling all the experimental condition and repetitions together. The amplitude and area of the MEPs were highly correlated (the average value \pm S.D. was $0.94 \pm 0.04$ ). For this reason, we report only the results of the amplitude and latency analysis. As expected, latencies for extrinsic hand muscles were markedly shorter than latencies of the intrinsic hand muscles (Table 1). The average difference was about $5 \mathrm{~ms}$, a time compatible with the transit time of the TMS-evoked descending volley along the peripheral nerve from the forearm to the hand. While the effects of initial grip force and TMS level on the latencies were often statistically significant due to the large number of data $(P<0.01$, two-way ANOVA for each muscle and subject, detailed results not reported to save place), the latencies varied by less than $1 \mathrm{~ms}$ across experimental conditions for a single muscle, making it physiologically almost irrelevant.

For all muscles and subjects, the amplitude increased with the level of TMS. On average the amplitude increased more or less linearly by $138 \%$ as the TMS increased by $33 \%$ (from MT to $133 \%$ of MT). The effect initial of grip force level depended on the muscle and on the subject but appeared to be more marked for the extrinsic than the intrinsic hand muscles (Fig. 9). Both factors and often the interaction were significant $(p<0.01$, detailed results of the two-way ANOVA of the MEP amplitude for each muscle and subject are not shown).

\subsection{Comparisons of MEFs and MEPs}

For each subject, we computed the coefficient of correlation for all possible pairs of MEP and MEF amplitudes (20 different coefficients of correlation can be computed by combining the four available MEPs with the five available MEFs, see Table 2). When data from all experimental conditions were pooled together (i.e., each coefficient of correlation was based on $N=192=16 \times 12$ data points), the coefficients of correlation were relatively large (average \pm S.D.: $0.64 \pm 0.16$ ). These positive correlations reflect the fact that the size of the MEFs and the amplitude of the MEPs tend to increase with TMS intensity and grip force level (see Figs. 6 and 9). In fact, the correlation coefficients were even higher $(0.79 \pm 0.12)$ when they were computed between the average values for each experimental condition 

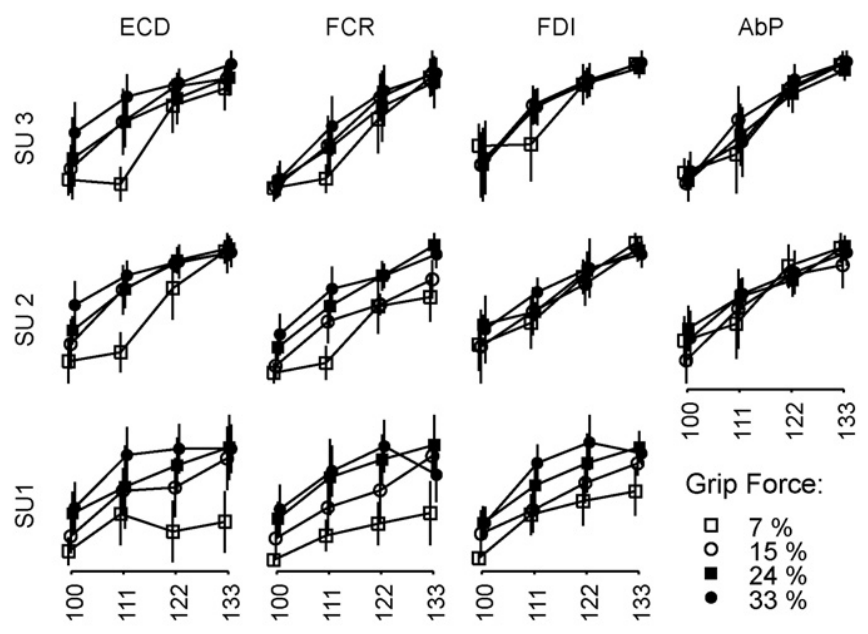

Grip Force:

ㅁ $7 \%$

- $15 \%$

- $24 \%$

TMS stimulation level (\% MT)

Fig. 9. MEP amplitudes as a function of the TMS intensity and initial level of grip force. Each panel plots the data from a particular muscle and subject (the AbP was not recorded for SU 1 and data for SU 4 were not analyzed for technical reasons, see Section 2). The vertical bars represent the standard errors $(N \leq 12)$. The symbols denote various levels of grip forces (see legend).

( $N=16$ data points per correlation of coefficient), i.e., when the across-repetition variability was removed. In contrast, the value of these coefficients of correlation was lower and more variable when they were computed inside each experimental condition $(0.41 \pm 0.37)$. The increased variability might be due in part to the fact that MEF values were often missing at low TMS intensities (see Fig. 2), which lowered the number of available data points and decreased the reliability of the estimates $(N=12$ data points per experiment condition and subject minus missing data points). In Fig. 10, a typical example of correlation between MEP and MEF is reported.

Table 2

Average values of the coefficients of correlation between MEP and MEP amplitudes

\begin{tabular}{cllllll}
\hline MEP & \multicolumn{7}{l}{ MEF } & \multirow{2}{*}{ Mean } \\
\cline { 2 - 5 } & Thumb & Index & Middle & Grip & Net & \\
\hline All repetition and conditions pooled & $(N=192)$ \\
ECD & 0.67 & 0.59 & 0.59 & 0.66 & 0.42 & 0.59 \\
FCR & 0.72 & 0.64 & 0.67 & 0.74 & 0.44 & 0.64 \\
FDI & 0.75 & 0.63 & 0.65 & 0.72 & 0.43 & 0.64 \\
AbP & 0.80 & 0.68 & 0.70 & 0.77 & 0.50 & 0.69 \\
Mean & 0.73 & 0.63 & 0.65 & 0.72 & 0.45 & 0.64
\end{tabular}

Correlation between average MEP and MEF amplitude, all conditions pooled $(N=16)$

$\begin{array}{lllllll}\text { ECD } & 0.86 & 0.76 & 0.77 & 0.83 & 0.62 & 0.77 \\ \text { FCR } & 0.89 & 0.80 & 0.82 & 0.87 & 0.62 & 0.80 \\ \text { FDI } & 0.90 & 0.79 & 0.78 & 0.85 & 0.68 & 0.80 \\ \text { AbP } & 0.92 & 0.79 & 0.79 & 0.86 & 0.68 & 0.81 \\ \text { Mean } & 0.89 & 0.79 & 0.79 & 0.85 & 0.65 & 0.79\end{array}$

Correlation inside each experimental condition $(N=12)$

\begin{tabular}{lllllll} 
ECD & 0.43 & 0.32 & 0.30 & 0.41 & 0.16 & 0.33 \\
FCR & 0.51 & 0.42 & 0.54 & 0.58 & 0.27 & 0.47 \\
FDI & 0.53 & 0.42 & 0.46 & 0.54 & 0.13 & 0.43 \\
AbP & 0.50 & 0.33 & 0.47 & 0.50 & 0.21 & 0.41 \\
Mean & 0.49 & 0.38 & 0.44 & 0.51 & 0.19 & 0.41 \\
\hline
\end{tabular}

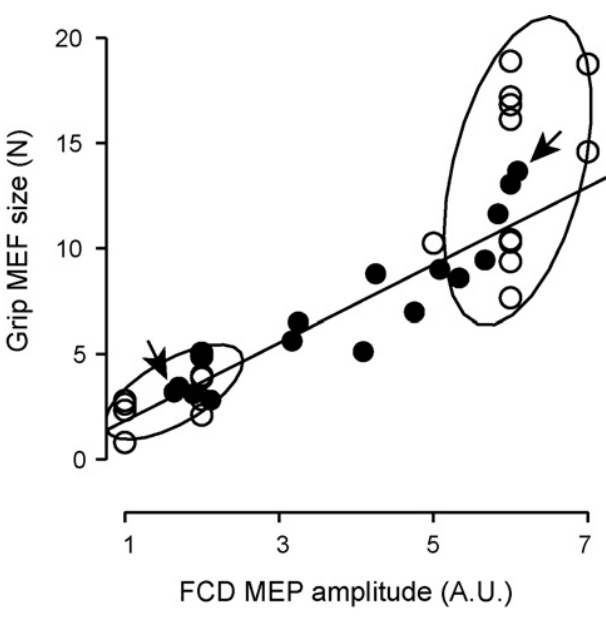

Fig. 10. This example of correlation between a MEPs and MEFs shows the amplitude of the MEF for the FCD muscle in abscissa and the MEF size for the grip force in ordinate. Empty points represent the values for each trial in two different experimental conditions: points on the left side of the plot correspond to the lowest TMS and initial grip force level (100\% of MT and 7\% of maximum grip force) while points on the right side correspond to the highest level (133\% of MT and $28 \%$ of maximum grip force. The coefficient of correlation for the values inside the two conditions was $r=0.66$ and 0.42 , respectively. The arrows designate the average value and center of the corresponding $68 \%$ ellipses of tolerance. Solid points represent the average values for each one of the 16 experimental conditions. The regression line is fitted to these average values (coefficient of correlation $r=0.93$ ).

When looking at specific MEP-MEF pairs (see Table 2), we found out that, for all muscles, the MEP amplitude was best correlated with the thumb or the grip $\mathrm{MEF}$, and least correlated with the net force. This finding held true whatever the way the coefficients of correlation were computed. It reflects the fact that the net force is in effect not directly related to the action of the single finger given that it depends essentially on the balance of forces between the thumb and the two opposing fingers. In contrast with the differences between the MEFs, the difference between MEPs were small. MEP of muscles that have a predominant mechanical effect on one finger did not appear to be significantly better correlated with the MEF of the corresponding finger.

\section{Discussion}

The main novelty of our study consisted in measuring complex "motor evoked forces" - such as the ones involved in human three-point grasping - following an external activation of the primary motor cortex by single-pulse TMS. The simultaneous recording of all finger forces is necessary to analyze the effect of the TMS at a level that is functionally meaningful and to compute the grip and net forces. As a matter of fact, both forces play an important role during the manipulation of objects: the task usually requires very precise control of the net force and grip force is an important index of grasp stability.

\subsection{Finger forces before TMS}

In our study, participants used visual feedback to maintain a zero net force while grasping a fixed object with various levels 
of grip force, an aspect of the task that has not yet been examined in the tripod grasp. In principle, two different synergies are compatible with the equilibrium conditions of the tripod grasp to maintain a null net force when increasing the grip force: the first one is a simple proportional increase of magnitudes of the three contact forces without directional shifts while the second one is a coordinated change in the finger force magnitude and direction.

We found that the pattern of forces used before the stimulation to grasp a fixed object was similar to the one used to lift an object. In both cases, the thumb force was directed toward a point approximately at mid-distance between the index and middle fingers $[2,48]$. In addition, our study shows that participants increased the grip force by increasing the finger forces proportionally, without changing their direction (Fig. 2). In other words, they used the first and simpler of the two aforementioned synergies.

\subsection{The main characteristics of MEFs}

The main finding of this study is that TMS produced synergistic changes of the finger forces. In particular, our study shows that MEFs during multi-finger grasps are to a large extent selfbalanced or, in other words, that the effect of TMS on the net force was quite small. As a matter of fact, it was impossible to identify an effect on the net force in about $35-40 \%$ of the trials for which an effect on the grip force could be identified. Moreover, the size of the MEF on the net force was smaller than the size of the finger MEFs or than the size of the grip MEF (Fig. 4). Finally, the increase of the finger forces (MEF size) was correlated across fingers for all subjects (Fig. 5).

A second noteworthy observation is the similarity existing between the TMS-induced responses and the voluntary increases of finger forces before TMS required by the task. As a matter of fact, we found that both the direction of the finger forces and the distribution of the grip force across fingers changed little as a result of the stimulation or when the subject increased the grip force (Table 1). This similarity suggests that TMS-induced responses use the same neurophysiological pathways as voluntary finger action, the idea being that the increase of finger forces would be proportional to the level of excitation of the corresponding neuronal pools. Moreover, the diminution of the MEF size expressed as a percentage of the finger force before the TMS with stronger initial levels of grip force (Fig. 6C) as well as the corresponding diminution of the effect of TMS on the direction of the finger forces (Fig. 7) suggests that neuronal pools that correspond to the ongoing prehension synergy become more stable when stronger forces are employed to grasp the object. This interpretation is also in line with the results of various studies that have shown that the amplitude of the MEPs [22] and MEFs $[9,30]$ depends the background activity of the corresponding muscles. As a matter of fact, the MEF sizes were correlated not only among themselves (Fig. 8A), but also with the background forces, at least at the highest level of TMS (Fig. 8B). This correlation pattern was most marked for the second participant, who also exhibited the largest net force before TMS (Fig. 2) and increase of net force (Fig. 4B). In that case, the increase of the net force might be simply viewed as an amplification of a pre-existing disequilibrium. It should however be noted that, in three out of four particpants, the background forces were not well correlated with the MEF sizes at the lowest level of stimulation, which indicate that other factors than the background force influenced the MEF sizes at these stimulation levels. While it is known that the type of the grasp (power or precision grasp) or the aperture in a precision grasp [23] can affect this relationship, this issue needs to be investigated further.

The effect on the TMS on the net force, while limited, cannot be completely neglected. In part, this effect can be seen, as noted above, as an amplification of a pre-existing disequilibrium. Another part of this effect could be due to a lack of complete coordination between the force magnitudes. As a matter of fact, the correlation between the MEF sizes, while highly significant, was not perfect. Variations of the force directions are not likely to have contributed in an important manner since both the systematic and random variations of the finger force directions decreased with a stronger grip force, as the net force increased. Moreover, the subject with the largest MEF angle (SU 4) was not the subject that produced the largest net force (SU 2 ). In the following section, we consider another possible cause of disequilibrium that is based on the observation of temporal asynchronies between the peak times of the MEFs.

\subsection{Temporal asynchronies}

The current study also allowed us to compare the latencies and peak time of the MEFs across different fingers. The first observation is an absence of significant differences in the latencies across fingers. In particular, the MEF for the thumb did not start before those of the other fingers, in spite of the fact that MEP of intrinsic muscles peaked later by about $5 \mathrm{~ms}$. The second and perhaps most striking observation is that the thumb MEF peaked, on average, $11 \mathrm{~ms}$ before the other fingers (i.e., the maximal peak force from the MEF onset was produced earlier than that of the other fingers). This asynchrony can possibly explain part of the increase of the net force observed in the last three subjects since the only participant with a very limited effect of TMS on the net force (SU 1) was also the only participant for which thumb force peaked at the same time as the other fingers (Fig. 4). Fig. 11 illustrates how different peak times across different fingers can create a temporary disequilibrium between the finger forces. In the first subject (SU 1, left panel), the effects of the TMS on the three finger forces were exactly balanced and the three finger forces peaked together with the net and grip forces. As a result, the net force remained close to zero throughout the trial (see inset). In contrast, for the other subject (SU 2), the thumb force peaked considerably later than the index or middle finger (107 ms vs. 73 and $83 \mathrm{~ms}$, respectively for this subject in this condition). As a result, the thumb force is initially insufficient to compensate for the increase of the index and middle fingers, causing net force in the same direction as these two fingers. The net force peaked $62 \mathrm{~ms}$ after the stimulation, which represented the moment at which the thumb force started to catch up with the index and middle finger forces. At the time of maximum grip force ( $82 \mathrm{~ms}$, see empty circle), the net force had already dimin- 
SU 1

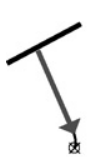

$2 \mathrm{~N}]$

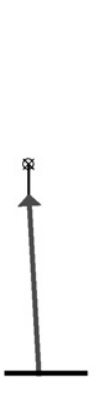

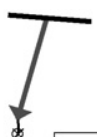

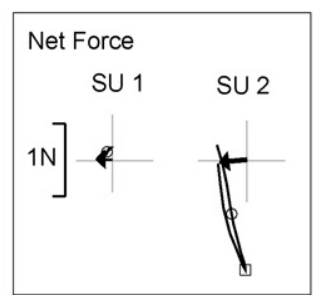

SU 2
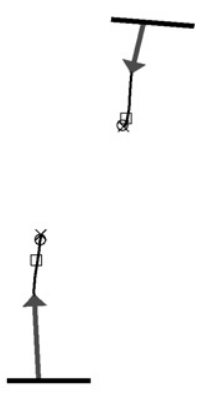

Fig. 11. MEFs for two different subjects with TMS set at $122 \%$ of MT and initial grip force level set at $15 \%$ of maximum grip force (across-repetitions average, $N=12$ ). The three arrows represent the finger forces at the time of the stimulation. The lines that start from the tip of the arrow denote the evolution of the finger forces from the stimulation time until peak time. The empty square and empty circle denote the position of the finger forces when the net and grip force peaked. The three short thick lines that are at the bases of the arrows represent the contact surfaces.

ished by half. Finally the net force had returned to its initial level once the thumb force peaked $(107 \mathrm{~ms})$. Thus, these data show that the lack of balance can be caused by differences in the rates with which the MEF reach their maximum values, even in the case where the forces do not change directions and the MEF sizes indicate a proportional increase of finger forces.

The difference in the peak time of the thumb and the other fingers cannot be explained by a corresponding shortening of the latency that remained constant and approximately equal for all fingers. A possible explanation of the difference in peak times is that different muscles have different electromechanical delay (EMD). As a matter of fact, the time from onset of EMG activity to change, as revealed by the MEP latency, to the realization of the resulting forces, as detected by the force sensor is know to vary in a considerably wide range of $20-130 \mathrm{~ms}$, depending on many muscular factors, such as length of the considered muscle, fiber types, strength, fatigue, training (see [50]). In the context of this study, EMD could also explain idiosyncratic differences across subjects, if one assumes that different motor synergies were used to produce the thumb force or that subjects assumed different hand postures. For example, the first subject might have a different mixture of synergistic muscle like flexor pollicis longus and the abductor pollicis to produce the thumb force. In this context, it might also be useful to recall that while the study also showed that large-scale change of MEF amplitude is accompanied by correspondingly large changes of MEP amplitudes, the values of the coefficients of correlation were low when we analyzed the relationship between MEF and MEP amplitude inside an experimental condition (Fig. 10), which suggests that different patterns of coordination between muscles were used to produce the MEF.

Moreover, variation in the EMD could also explain the shortening of the peak times observed in all MEFs higher initial level of grip force. As a matter of fact, increase of background activity tends to decrease the EMD [50]. In this case, the variations of the EMD might also be caused by an increase of the excitability at the cortical or spinal level when the finger forces are stronger,

as reflected by the corresponding increase of the MEPs amplitude in several subjects and muscles (Fig. 9). Further research is needed to assess if such peripheral factors can explain the observed temporal asynchronies or if central factors are also at play.

\subsection{MEFs and motor synergies}

It has been suggested that, during voluntary movements, the CNS selectively restricts solutions that lead to desired values of important control variables while making use of the abundant solutions available to it by allowing a range of functionally equivalent solutions to be instantiated, the exact solution dependent on momentary fluctuations of local dynamics and external constraints (the uncontrolled manifold [UCM] hypothesis; [46]). For example, in a force production task where the important task variable is the total force output, the UCM approach explains well the observed covariation patterns of finger forces that correspond to different ways of distributing the total force across fingers [45]. In the context of our study, if we assume that TMSinduced responses use the same neurophysiological pathways as voluntary finger action, the UCM hypothesis predicts that the effect of the TMS should be less marked along the dimensions that corresponds to the task variables, i.e., the net and grip forces, because the CNS would stabilize the variations along the dimensions that are relevant to the task when confronted with the problem of selecting a solution. The results of our study are however difficult to explain in this framework. In particular, the TMS did not cause changes in the direction of the finger forces and/or the distribution of the grip force across fingers that were compatible with the task constraints. Instead, the TMS had a marked effect on the grip force, a variable that participants were explicitly asked to maintain during the experiment.

These observations clearly suggest that the grip and net force have a different status, independently of the task constraints. They also raise the question of whether the grip and net forces are governed by different neurophysiological mechanisms. As a matter of fact, various studies have considered the possibility that contact forces can be decomposed in a grasping and manipulative component, each being controlled independently as in some robotic manipulators (e.g., $[55,17,48]$ ). In the context of our study, the idea is that areas upstream with respect to the point of stimulation would control the manipulative component and maintain the motor cortex in a dynamic state whereby the finger forces produced the desired net force. A similar hypothesis has been proposed to explain the enslaving effect, i.e., the tendency to produce force by non-instructed finger in a multi-finger force production task [9]. In Danion et al.'s study, the MEF sizes depended on the background force of the corresponding finger and not on the background force of other fingers, as one might expect if physiologically independent neuronal subpools served individual digits. In other words, this finding was interpreted as evidence that the neurophysiological substrate of the enslaving effect lies in brain structures that project to the motor cortex. In the context of our study, the idea is that stimulation of the motor cortex would simply increase the activity of currently activated pools of neurons without modifying the prehension synergy, 
thereby increasing the grip force without affecting the net force, at least at the lower levels of stimulation. Admittedly, evidence for such a control scheme is currently at best mixed. On the one hand, the fact that one can easily vary the internal forces of the grasp without moving the object supports this idea. For example, the distribution of the normal forces across fingers opposing the thumb in the prismatic grasp [47] or the direction of the finger force in the tripod grasp (Baud-Bovy, unpublished) can also be varied without affecting the net force or moment. On the other hand, there is a well-established coupling between the grip force and the load force in the pinch grasp, when the load (gravitational or inertial forces) is perpendicular to the line defined by the contact points that define the grip force [27]. Such a coupling has also been evidenced in multi-fingered grasps when the load acted in a direction that was orthogonal to the null space of the grasp [17]. The grasping force was also found to covary with the load when the net force was not orthogonal to the null space of the grasp but, in this case, the role of the CNS is more difficult to assess because variations of the internal forces can at least in part be ascribed to the visco-elastic properties of the fingers [48].

To conclude, this study shows the potential of the TMS and the MEFs to investigate the neurophysiological processes underlying the functional synergies involved in a complex motor skill such as grasping. In addition, if our interpretation is correct, the effect of the TMS on the net force could be used to assess the stability of prehension synergies in a variety of conditions, both with intact humans and brain-damaged patients. Future research is needed however to quantify more precisely the role of the various factors that might have contributed to the presence of a residual effect of TMS on the net force. In particular, it might be useful to record MEPs from a larger number of muscles to investigate the relative contribution of central and peripheral factors to the MEFs. Finally, future studies should also examine if the TMS continues to evoke synergistic finger forces in different tasks and/or non-static conditions.

\section{References}

[1] A.T. Barker, R. Jalinous, I.L. Freeston, Non invasive magnetic stimulation of the human motor cortex, Lancet 1 (1985) 1106-1107.

[2] G. Baud-Bovy, J.F. Soechting, Two virtual fingers in the control of the tripod grasp, J. Neurophysiol. 86 (2001) 604-615.

[3] G. Baud-Bovy, J.F. Soechting, Factors influencing variability of load forces in a tripod grasp, Exp. Brain Res. 143 (2002) 57-66.

[4] G. Baud-Bovy, D. Prattichizzo, N. Broggi, Does torque minimization yield a stable grasp? in: F. Barbagli, D. Prattichizzo, K. Salisbury (Eds.), Multi-Point Interaction in Robotics and Virtual Reality. Springer Tracts for Advanced Robotic (STAR), Springer, Berlin, 2005, pp. 21-40.

[5] N.A. Bernstein, On dexterity and its development, 1967, in: M.L. Latash, M.T. Turvey (Eds.), Dexterity and Its Development, Lawrence Erlbaum Associates, 1996.

[6] U. Castiello, The neuroscience of grasping Nat. Rev. Neurosci. 6 (2005) 726-736.

[7] L. Cattaneo, M. Voss, T. Brochier, G. Prabhu, D.M. Wolpert, R.N. Lemon, A cortico-cortical mechanism mediating object-driven grasp in humans, Proc. Natl. Acad. Sci. USA 102 (2005) 898-903.

[8] P.A. Chouinard, G. Leonard, T. Paus, Role of the primary motor and dorsal premotor cortices in the anticipation of forces during object lifting, J. Neurosci. 25 (9) (2005) 2277-2284.
[9] F. Danion, M.L. Latash, S. Li, Finger interactions studied with transcranial magnetic stimulation during multi-finger force production tasks, Clin. Neurophysiol. 114 (2003) 1445-1455.

[10] M. Davare, M. Andres, G. Cosnard, J.-L. Thonnard, E. Olivier, Dissociating the role of ventral and dorsal premotor cortex in precision grasping, J. Neurosci. 26 (8) (2006) 2260-2268.

[11] M. Davare, M. Andres, E. Clerget, J.-L. Thonnard, E. Olivier, Temporal dissociation between hand shaping and grip force scaling in the anterior intraparietal area, J. Neurosci. 27 (15) (2007) 3974-3980.

[12] M. Davare, J. Duque, Y. Vandermeeren, J.-L. Thonnard, E. Olivier, Role of the ipsilateral primary motor cortex in controlling the timing of hand muscle recruitment, Cereb. Cortex 17 (2007) 353-362.

[13] V. Di Lazzaro, A. Oliviero, P. Profice, E. Saturno, F. Pilato, A. Insola, P. Mazzone, P. Tonali, J.C. Rothwell, Comparison of descending volleys evoked by transcranial magnetic and electric stimulation in conscious humans, Electroenceph. Clin. Neurophysiol. 109 (1998) 397-401.

[14] H.H. Ehrsson, A. Fagergren, T. Jonsson, G. Westling, R.S. Johansson, H. Forssberg, Cortical activity in precision-versus power-grip tasks: an fMRI study, J. Neurophysiol. 83 (2000) 528-536.

[15] H.H. Ehrsson, A. Fagergren, H. Forssberg, Differential fronto-parietal activation depending on force used in a precision grip task: an fMRI study, J. Neurophysiol. 85 (2001) 2613-2623.

[16] H.H. Ehrsson, A. Fagergren, R.S. Johansson, H. Forssberg, Evidence for the involvement of the posterior parietal cortex in coordination of fingertip forces for grasp stability in manipulation, J. Neurophysiol. 90 (2003) 2978-2986.

[17] F. Gao, M.L. Latash, V.M. Zatsiorsky, Internal forces during object manipulation, Exp. Brain Res. 165 (1) (2005) 69-83.

[18] A.P. Georgopoulos, J. Ashe, N. Smyrnis, M. Taira, The motor cortex and the coding of force, Science 256 (5064) (1992) 1692-1695.

[19] C.C.A.M. Gielen, B.M. van Bolhuis, M. Theeuwen, On the control of biologically and kinematically redundant manipulators, Hum. Mov. Sci. 14 (4-5) (1995) 487-509.

[20] M. Graziano, The organization of behavioral repertoire in motor cortex, Ann. Rev. Neurosci. 29 (2006) 105-134.

[21] M. Hallett, Transcranial magnetic stimulation: a primer, Neuron 55 (2007) 187-199.

[22] Y. Hasegawa, T. Kasai, T. Tsuji, S. Yahagi, Further insight into the task-dependent excitability of motor evoked potentials in first dorsal interosseous muscle in humans, Exp. Brain Res. 4 (2001) 387-396.

[23] Y. Hasegawa, T. Kasaia, H. Kinoshitab, S. Yahagic, Modulation of a motor evoked response to transcranial magnetic stimulation by the activity level of the first dorsal interosseous muscle in humans when grasping a stationary object with different grip widths, Neurosci. Lett. 299 (2001) 1-4.

[24] M.-C. Hepp-Reymond, M. Kirkpatrick-Tanner, L. Gabernet, H.-X. Qi, B. Weber, Context-dependent force coding in motor and premotor cortical areas, Exp. Brain Res. 128 (1999) 123-133.

[25] C.W. Hess, K.R. Mills, N.M.F. Murray, Responses in small hand muscles from magnetic stimulation of the human brain, J. Physiol. 388 (1987) 397-419.

[26] M. Jeannerod, M.A. Arbib, G. Rizzolatti, H. Sakata, Grasping objects: the cortical mechanisms of visuomotor transformation, Trends Neurosci. 18 (1995) 314-320.

[27] R.S. Johansson, K.J. Cole, Grasp stability during manipulative actions, Can. J. Physiol. Pharmacol. 72 (1994) 511-524.

[28] R.S. Johansson, R.N. Lemon, G. Westling, Time-varying enhancement of human cortical excitability mediated by cutaneous inputs during precision grip, J. Physiol. 181 (3) (1994) 761-775.

[29] J.P. Kuhtz-Buschbeck, H.H. Ehrsson, H. Forssberg, Human brain activity in the control of fine static precision grip forces: an fMRI study, Eur. J. Neurosci. 14 (2001) 382-390.

[30] M.L. Latash, K. Yarrow, J.C. Rothwell, Changes in finger coordination and responses to single pulse TMS of motor cortex during practice of a multifinger force production task, Exp. Brain Res. 151 (1) (2003) 60-71.

[31] R.N. Lemon, R.S. Johansson, G. Westling, Corticospinal control during reach, grasp, and precision lift in man, J. Neurosci. 15 (1995) $6145-6156$. 
[32] M.M. Morrow, L.R. Jordan, L.E. Miller, Direct comparison of the task-dependent discharge of M1 in Hand space and muscle space, J. Neurophysiol. 97 (2007) 1786-1798.

[33] Z. Ni, N. Liang, M. Takahashi, T. Yamashita, S. Yahagi, Y. Tanaka, T. Tsuji, T. Kasai, Motor strategies and excitability changes of human hand motor area are dependent on different voluntary drives, Eur. J. Neurosci. 12 (2006) 3399-3406.

[34] D.A. Nowak, M. Voss, Y.-Z. Huang, D.M. Wolpert, J.C. Rothwell, Highfrequency repetitive transcranial magnetic stimulation over the hand area of the primary motor cortex disturbs predictive grip force scaling, Eur. J. Neurosci. 22 (2005) 2392-2396.

[35] G. Prabhu, M. Voss, T. Brochier, L. Cattaneo, P. Haggard, R. Lemon, Excitability of human motor cortex inputs prior to grasp, J. Physiol. 581 (2007) 189-201.

[36] S. Rossi, P.M. Rossini, TMS in cognitive plasticity and the potential for rehabilitation, Trends Cogn. Sci. 8 (2004) 271-279.

[37] P.M. Rossini, S. Rossi, Transcranial magnetic stimulation: diagnostic, therapeutic, and research potential, Neurology 68 (2007) 484-488.

[38] P.M. Rossini, A.T. Barker, A. Berardelli, et al., Non-invasive electrical and magnetic stimulation of the brain spinal cord and roots) basic principles and procedures for routine clinical application, Electroencephalogr. Clin. Neurophysiol. 91 (1994) 79-92.

[39] P.M. Rossini, M.D. Caramia, C. Iani, M.T. Desiato, G. Sciarretta, G. Bernardi, Magnetic transcranial stimulation in healthy humans: influence on the behavior of upper limb motor units, Brain Res. 676 (1995)314-324.

[40] P.M. Rossini, S. Rossi, P. Pasqualetti, F. Tecchio, Corticospinal excitability modulation to hand muscles during movement imagery, Cereb. Cortex 9 (1999) 161-167.

[41] J.K. Salisbury, B. Roth, Kinematic and force analysis of articulated mechanical hands, ASME J. Mech., Transm. Actuation Des. (presently known as the J. Mech. Des.) 105 (1983) 35-41.

[42] M.H. Schieber, Constraints on somatotopic organization in the primary motor cortex, J. Neurophysiol. 86 (2001) 2125-2143.
[43] M.H. Schieber, M. Santello, Hand function: peripheral and central constraints on performance, J. Appl. Physiol. 96 (2004) 2293-2300.

[44] M. Schieppati, C. Trompetto, G. Abbruzzese, Selective facilitation of responses to cortical stimulation of proximal and distal arm muscles by precision tasks in man, J. Physiol. (Lond.) 491 (1996) 551-562.

[45] J.P. Scholz, F. Danion, M.L. Latash, G. Schöner, Understanding finger coordination through analysis of the structure of force variability, Biol. Cybern. 86 (2002) 29-39.

[46] G. Schöner, Recent developments and problems in human movement science and their coceptual implications, Ecol. Psychol. 8 (1995) 291-314.

[47] J.K. Shim, M.L. Latash, V.M. Zatsiorsky, Prehension strategies: trial-totrial variability a hierarchical organization of stable performance, Exp. Brain Res. 152 (2003) 173-184.

[48] M.A. Smith, J.F. Soechting, Modulation of grasping forces during object transport, J. Neurophysiol. 93 (2005) 137-145.

[49] E. Todorov, M.I. Jordan, Optimal feedback control as a theory of motor coordination, Nat. Neurosci. 5 (11) (2002) 1226-1235.

[50] P.F. Vint, S.P. McLean, G.M. Harron, Electromechanical delay in isometric actions initiated from non-resting levels, Med. Sci. Sport Exerc. 33 (2001) 978-983.

[51] E.M. Wassermann, J.M. Tormos, A. Pascual-Leone, Finger movements induced by transcranial magnetic stimulation change with hand posture, but not with coil position, Hum. Brain Mapp. 6 (1998) 390-393.

[52] T. Yoshikawa, K. Nagai, Manipulating and grasping forces in manipulation by multifingered robot hands, IEEE Trans. Robotics Automation 7 (1) (1991) 67-77.

[53] V.M. Zatsiorsky, M.L. Latash, Prehension Synergies, Exerc. Sport Sci. Revi. 32 (2) (2004) 75-80.

[54] V.M. Zatsiorsky, M.L. Latash, F. Gao, J.K. Shim, The principle of superposition in human prehension, Robotica 22 (2004) 231-234.

[55] B.R. Zuo, W.H. Qian, A general dynamic force distribution algorithm for multi-fingered grasping, IEEE Trans. Syst. Man. Cybern. 30 (2000) 185-192. 